\title{
A Novel Wind Power Capacity Combined Forecasting method based on Backtracking Search Algorithm

\author{
JunChao Yang
}

School of Economic and Management Administration, North China Electric Power University, Beijing, 102206, China

email: gs_windboy@126.com

Keywords: Wind capacity forecasting; Differential evolution algorithm; support vector regression

\begin{abstract}
As wind power is a mature and important renewable energy, wind power capacity forecasting plays an important role in renewable energy generation's plan, investment and operation. Combined model is an effective load forecasting method; however, how to determine the weights is a hot issue. This paper proposed a combined model with backtracking search algorithm for optimizing weights, which can improve the performance of each single forecasting model of regression, BPNN and SVM. In order to prove the effectiveness of the proposed model, an application of the China's wind power capacity from 2001 to 2013 was evaluated. The experiment results show that the proposed model gets the maximum mean absolute percentage error (MAPE) value $4.72 \%$, which is better than the results of regression, BPNN and SVM.
\end{abstract}

\section{Introduction}

Recently, greenhouse gas emission (GHG), especially CO2 emission, as a serious environment problem has attracted the attention of the world. CO2 emission will cause the greenhouse effect, which will cause global warming by IPCC's report, and change the climatic characteristics of the world. Due to electricity power industry is one of the largest departments of a nation's CO2 emission, reducing CO2 emissions of electricity power industry is very significant of a nation. Renewable energy generation is an important way for electricity power industry to achieve the energy saving goal, which helps China finish the promise of decreasing carbon emissions per unit of GDP by $40-45 \%$ in 2020 compared with 2005. As wind power generation is the most mature renewable energy technology in the renewable energy generation, it has been developed rapidly. From 2000 to 2008, the increasing rate of wind power generation in main developed country has been improved more than $20 \%$.

Many techniques have been proposed for wind power forecasting such as wind speed forecasting and wind power forecasting, and the forecasting methods include the autoregressive model, ARIMA[1] model, ANN model[2-3], GMDH model[4] and so on. It regrets that few researches pay attention to the forecasting problem about wind power capacity, which plays an important role for the wind power construction plan, investment and operation. And these papers are all focused on using a single model to solve the wind power forecasting, some forecasting scholars have been pointed out that a single model's performance is worse than combined models'. The reasons are as follows, first, the combined model can reduce disadvantage of any a single model; second, the errors of combined model are always smaller than each single model's. Therefore, this paper proposed a combined forecasting model of wind power capacity with differential evolution algorithm to solve the problem.

\section{A combined forecasting model with backtracking search algorithm}

In practice, how to choose the best forecasting model is a difficult problem, because a load forecasting problem can use different load forecasting models, and the load forecasting accuracy are also different. In above studies, the mentioned load forecasting models are focused to use one excellent model to solve a load forecasting problem, it will even cause solving the same type load forecasting problem by different forecasting methods. So some scholars present to use a combined 
model, which is combining several forecasting models to solve a forecasting problem. The reasons are shown as follows:

(1) The combined model can own the advantages of each single forecasting model, and it also can reduce the disadvantages of single model;

(2) The load forecasting accuracy of combined model always smaller than it of a single forecasting model comparing with a single forecasting model,

(3) It brings a various result of middle-long forecasting, which can give more information of decision-making. To use a combined forecasting model, a key problem is to determine the weight of each forecasting models. Therefore, this paper proposed a hybrid model which is using differential evolution algorithm to determine the weights of the combined forecasting model.

A combined forecasting model is consist of two or more forecasting models and each model has a certain weights, which can be described as :Suppose that $f_{i}$ is forecasting results of the ith method, $y$ is the actual data. The combined forecasting model $\hat{y}$ can be expressed by

$$
\hat{y}=\sum_{i=1}^{n} w_{i} f_{i}
$$

In which, $w_{i}$ is the weight of the ith method, and an optimization problem of the combined model need satisfy the following model.

$$
\begin{aligned}
& \operatorname{Min}: \operatorname{MSE}\left(y-\sum_{i=1}^{n} w_{i} f_{i}\right) \\
& \text { In which, s.t. } \sum_{i=1}^{n} w_{i}=1 ; w_{i} \geq 0
\end{aligned}
$$

The key problem of the combined model is to determine the weight. The weight determine methods can divide into two categories, one is fixed weight method, which determined the weight as a fixed number, and the other is dynamic weight method, which determined the weight as a function of the time. The fixed weight determined model is the most popular method because it is simple and easy to apply, and it is suitable for using intelligence algorithm such as genetic algorithm, particle swarm optimization to solve the problem.

Backtracking Search Algorithm (BSA) algorithm is one of the evolutionary algorithms(EA), which was proposed by Pinar Civicioglu in 2013. As other evolution algorithms such as genetic algorithm (GA), BSA is also population based algorithm, which contains five steps as is done in other EAs: initialization, selection-I, mutation, crossover and selection-II. The detail working steps are as follows[5]:

Step 1.Parameters initialization

The main parameters of BSA algorithm are population size $N$ and length of the chromosome, which is always the problem dimension $D$, Set $g=0$. Generate a $N^{*} D$ matrix with uniform probability distribution random values. The generation method is

$$
X_{i j}=\operatorname{rand} \cdot(\operatorname{high}[j]-\operatorname{low}[j])+\operatorname{low}[j]
$$

In which, $i=1,2, \ldots, N, j=1,2, \ldots, D$,rand is a random number with a uniform probability distribution, and high[j], low $[j]$ is the upper bound and lower bound of the $j$ th column, respectively.

\section{Step 2. Selection-I}

In this stage, BSA determines which population is to be used for calculating the search direction, the population is determined using Eq. (4), and $U$ is the uniform distribution:

oldP $_{i j} \sim \mathrm{U}\left(\operatorname{low}_{\mathrm{j}}, \mathrm{up}_{\mathrm{j}}\right)$

at the beginning, BSA has the option of redefining oldP of each iteration through the IF-THEN rule by Eq. (5):

if $a<b$ then oldP $:=P \mid a, b \sim U(0,1)$

where := is the update operation. After oldP is determined, Eq. (4) is used to randomly change the order of the individuals in oldP:

oldP := permuting $($ old $P)$

The permuting function used in Eq. (6) is a random shuffling function. 
Step 3. Mutation operation

BSA generates the trial population using mutation function as Eq. (7).

$$
\text { Mutant }=P+F *(\text { old } P-P)
$$

In Eq. (7), F controls the amplitude of the search-direction matrix (oldP - P). Because the historical population is used in the calculation of the search-direction matrix, BSA generates a trial population, taking partial advantage of its experiences from previous generations. This paper uses $\mathrm{F}$ $=3 *$ rndn, where rndn N(0,1).

Step 4. Crossover operation

BSA's crossover process generates the final form of the trial population $T$. The initial value of the trial population is Mutant, as set in the mutation process. Trial individuals with better fitness values for the optimization problem are used to evolve the target population individuals. BSA's crossover process has two steps. The first step calculates a binary integer-valued matrix of size $\mathrm{N} * \mathrm{D}$ that indicates the individuals of $T$ to be manipulated by using the relevant individuals of $P$.

Step 5.Selection-II operation

Selection-II operation $T_{i}$ S that have better fitness values than the corresponding $P_{i} S$ are used to update the $P_{i} s$ based on a greedy selection. If the best individual Pbest has a better fitness value than the global minimum value obtained so far by BSA, the global minimizer is updated to be Pbest, and the global minimum value is updated to be the fitness value of Pbest . the algorithm and the source code of BSA can be gotten in [20].

BSA algorithm has the better performance than PSO, CMAES, ABC, JDE, CLPSO and SADE algorithms in the examination of several widely used benchmark functions[ref]. According to the study, BSA algorithm is stable and it can obtain a better solution than other algorithms, and the experiment results are also shown that BSA can get the nearest optimal solution than PSO and GA algorithm. Therefore, the BSA algorithm will use to solve the weight determining optimization problem, whose fitness function is employed the mean absolute percentage error function (MAPE), which is common using in load forecasting performance evaluation[21-24].

$$
M A P E=\frac{1}{n} \sum_{i=1}^{n}\left|\frac{A(i)-F(i)}{A(i)}\right| \times 100 \%
$$

Where $A(i)$ is the actual value, $F(i)$ is the forecasting value and $n$ is the total numbers.

\section{A numeric example}

In this case, we use the annual wind power capacity of China from 2001 to 2013, With China's economic rapid development, the load curve appears an exponential curve character, so we are using the $\ln (\mathrm{x})$ value as the dependent variable in the single forecasting models. And the single forecasting models are chosen as regression model, back propagation neural network(BPNN) and support vector machine(SVM) models, because these models are all the popular single models in load forecasting.

In BPNN forecasting model, the node number of input layer is $x t-3, x t-2, x t-1$, the hidden layer number is five and the output node is Xt . In SVM forecasting model, the input variables are also $\mathrm{xt}-3$, xt-2, xt-1, and the output node is also xt, the same as BPNN model, and the parameters is the default in LIBSVM. The single forecasting results are shown in Table1. Because the accuracy form 2001-2006 are unsatisfactory, we only use the result from 2007 to 2013 for combining and comparing. As a result, the proposed combined weight of regression, BPNN and SVM, which determined by BSA algorithm, are $0.2469,0.1000$ and 0.6705 respectively. The final results are all shown in Table 1 and Fig. 1.

From the determined weight of BSA algorithm, it can be clearly seen that BPNN model get the maximum weight, SVM follows, and the regression model's weight is almost zero. The better the performance is, the greater the weight is. It proves that BSA algorithm is effective. 
Table 1. The actual load and forecasting results of regression, ANN, SVM and combined model

\begin{tabular}{cccccc} 
Year & Actual load & Regression & BPNN & SVM & Combined Model \\
\hline 2007 & 5848 & 6417.1 & 4665.3 & 5656.6 & 5743.7 \\
2008 & 12002 & 10456.5 & 12506.4 & 13270.1 & 12512.3 \\
2009 & 25805 & 17964.4 & 23643.5 & 26689.0 & 24272.7 \\
2010 & 44734 & 34804.1 & 31783.0 & 37892.0 & 36542.3 \\
2011 & 62364 & 57897.4 & 58234.0 & 64296.9 & 62148.2 \\
2012 & 75324 & 79406.0 & 79330.0 & 76605.6 & 77552.9 \\
2013 & 91413 & 95217.2 & 92473.0 & 89297.1 & 91045.7 \\
MAPE & -- & $13.13 \%$ & $10.69 \%$ & $5.67 \%$ & $4.72 \%$ \\
\hline
\end{tabular}

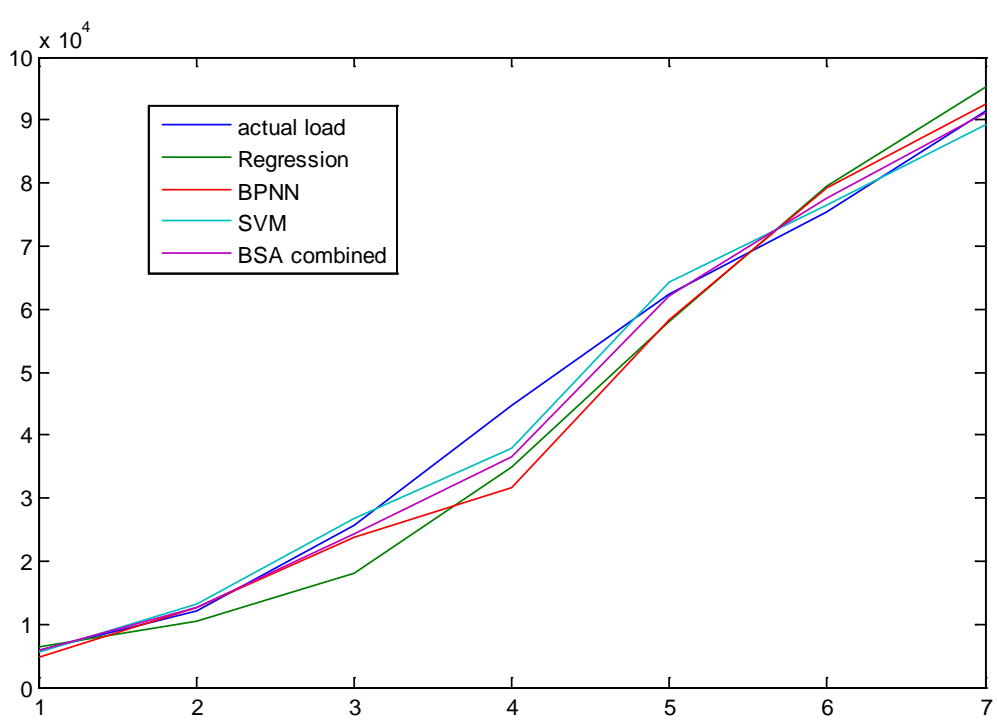

Fig. 1. The forecasting results of regression, ANN, SVM and combined model.

\section{Conclusion}

The proposed combined model integrates the regression, BPNN and SVM models. With using BSA algorithm to determine the weight, it can improve the load forecasting performance. In the experiment, the proposed method gets the minimum MAPE values, which verifies the proposed method's effectiveness in wind power capacity forecasting.

\section{References}

[1] Erdem E, Shi J. ARMA based approaches for forecasting the tuple of wind speed and direction[J]. Appl Energy 201188 1405-1414.

[2] Monfared M, H. Rastegar H, Kojabadi HM. A new strategy for wind speed forecasting using artificial intelligent methods[J]. Renewe Energy 200934 845-848.

[3] Guo ZH, Zhao WG, Lu HV, Wang JZ. Multi-step forecasting for wind speed using a modified EMD-based artificial neural network model[J]. Renew Energy 201237 241-249.

[4] Abdel-Aal RE, Elhadidy MA, Shaahid SM. Modeling and forecasting the mean hourly wind speed time series using GMDH-based abductive networks[J]. Renew Energy 200934 1686-1699.

[5] Pinar Civicioglu. Backtracking Search Optimization Algorithm for numerical optimization problems[J]. Applied Mathematics and Computation. 2013219 8121-8144. 